\title{
A supervisáo clínico-institucional como dispositivo de qualificação na Atenção Psicossocial: uma experiência de parceria com a Universidade
}

\author{
I ${ }^{1}$ Claudia Henschel de Lima, ${ }^{2}$ Thalles Cavalcanti dos Santos Mendonça Sampaio, \\ 3 Julia da Silva Cunha I
}

Resumo: Apresenta-se a condução da supervisão clínico-institucional em um centro de atenção psicossocial localizado no município de Volta Redonda, no estado do Rio de Janeiro, resultando de projeto de extensão que vigorou entre os anos de 2012 e 2015, em uma universidade localizada no mesmo município. O processo de reforma psiquiátrica no município é apresentado a partir de duas interrogaçóes do projeto de extensão: Em que momento da reforma psiquiátrica se encontra o município de Volta Redonda? Que clínica fora possível, até aquele momento, no CAPS? A partir dessas interrogaçóes, descreveu-se o processo da supervisão clínico-institucional, destacando a psicanálise como marco teórico principal na análise sobre a forma como o cotidiano dos usuários do CAPSII se situa no horizonte da reforma psiquiátrica, sobre o papel da psiquiatria no CAPS e na construção de um saber alternativo à psiquiatria, que considere a relevância da teoria, da posição subjetiva dos usuários e do saber dos profissionais. Destaca-se, ao final, a relevância da parceria com a universidade, como espaço de formação e reflexão crítica permanente sobre a oferta de serviços em saúde mental no horizonte da reforma psiquiátrica.

> Palavras-chave: supervisão clínico-institucional; atenção psicossocial; universidade; Reforma Psiquiátrica; psicanálise.

\author{
1 Programa de Pós-Graduação \\ em Psicologia, Universidade \\ Federal do Rio de Janeiro. Rio de \\ Janeiro-RJ, Brasil (claudiahlima@ \\ yahoo.com.br). \\ ORCID: 0000-0002-7693-7321. \\ 2 Psicologia, Universidade Federal \\ Fluminense. Niterói-RJ, Brasil \\ (thallesampaio@msn.com). \\ ORCID: 0000-0002-5527-7774. \\ ${ }^{3}$ Instituto de Ciências Humanas \\ e Filosofia. Universidade Federal \\ Fluminense. Niterói-RJ, Brasil \\ (jpatysc@hotmail.com). \\ ORCID: 0000-0002-4068-182X
}

Recebido em: 30/04/2018 Revisado em: 03/03/2019 Aprovado em: 12/03/2019 


\section{Introdução}

$\mathrm{O}$ artigo apresenta o processo de condução da supervisão clínico-institucional (SCI) em um centro de atençâo psicossocial, localizado no município de Volta Redonda (Regiáo Sul do Estado do Rio de Janeiro). A supervisão clínico-institucional (SCI) resultou do projeto de extensão que vigorou entre os anos de 2012 e 2015, sob a responsabilidade de uma das autoras, que atua como docente e pesquisadora de uma universidade pública, localizada no mesmo município. O projeto esteve articulado aos eixos de ensino e pesquisa e com concentração em saúde e direitos humanos e reconhece a importância de aproximar a universidade dos problemas e necessidades do município, em especial no que se referia à área da saúde mental. Seu objetivo foi a elaboração do programa de formação e qualificação dos profissionais da rede de saúde mental de Volta Redonda, sustentado nos seguintes eixos:

1. A recuperação do processo de Reforma Psiquiátrica no Brasil, e que resultou na promulgação da Lei n. 10.216/2001. E, mais precisamente, a especificação desse processo no município, culminando com a intervenção do poder público municipal na Casa de Saúde de Volta Redonda (CSVR) no ano de 1994, até a criação do primeiro Centro de Atenção Psicossocial na cidade de Volta Redonda (CAPS Usina dos Sonhos).

2. A elaboração, com base na articulação conceitual entre psicanálise e saúde mental, da SCI no CAPS II - Dr. Sergio Sibilio Fritsch (bairro Jardim Belvedere), para acolhimento de formas de sofrimento psíquico grave e persistente em adultos.

O eixo de sustentação da Reforma Psiquiátrica foi a substituição do modelo hospitalocêntrico - que previa a internação e o isolamento social como diretrizes de tratamento - pelo modelo de atenção psicossocial na saúde mental, centrado no usuário em sofrimento psíquico e na importância do laço social tanto sobre o desencadeamento como sobre seu processo de estabilização. A centralidade no usuário foi situada como diretamente proporcional à descentralização da psiquiatria, implicando a modificação na relação entre a equipe responsável por seu acolhimento e abordagem e o sofrimento psíquico que o atravessa: a atenção psicossocial não concentra um princípio normativo de direção de tratamento em torno da autoridade do psiquiatra. E a SCI exerceu, no quadro do modelo de atenção psicossocial, 
um lugar central em sua consolidação. Seu estabelecimento se deu por meio da portaria 1.174/2005, a partir de uma deliberação proveniente do I Congresso Brasileiro de Centros de Atenção Psicossocial, em 2004, sobre a relevância da SCI na formação e qualificação dos profissionais dos Centros de Atenção Psicossocial e, consequentemente, sobre a garantia de sua regularidade. A defesa dessa relevância respondia ao entendimento de que a supervisão, por ser clínico-institucional, conferiria aos profissionais da atenção psicossocial um modo de abordagem do sofrimento psíquico distinto da compreensão especializada e ordenada em torno do saber psiquiátrico típica do modelo hospitalocêntrico. De fato, ao posicionar os casos clínicos em articulação com o contexto institucional - composto pelo próprio serviço, pela rede, gestão e a implantação de política pública de saúde mental -, a SCI viabilizaria aos profissionais da atenção psicossocial uma nova concepção acerca:

1. Do sofrimento psíquico em articulação direta com a dimensão política.

2. Da gestão em saúde mental, com a valorização do compartilhamento dos níveis de conhecimento teórico e manejo técnico imanente à formação de cada profissional da equipe e à pactuação de responsabilidades, assegurando a circulação democrática do saber e rompendo com a rigidez das especialidades características do modelo hospitalocêntrico.

3. Dos procedimentos de intervenção clínica a partir da relevância atribuída aos marcos teóricos em psicopatologia na precisão da escuta do sofrimento psíquico e da identificaçáo dos fatores sociais que concorrem para seu desencadeamento.

O quadro atual da política para a saúde mental no Brasil vem propiciando a revalorização do modelo hospitalocêntrico, tanto pela inserção dos hospitais psiquiátricos na Rede de Atenção Psicossocial (RAPS) - conforme a recomendação, pela Coordenaçáo Nacional de Saúde Mental - como pelo aumento do montante de investimento, desde 2017, para os hospitais psiquiátricos. Isso tem se refletido, diretamente, na rotina de funcionamento da atenção psicossocial no Brasil: limitação de investimentos, fragilização da RAPS, relação com os gestores em um quadro de austeridade no investimento, precarização da formação permanente dos profissionais que integram os diversos equipamentos de saúde mental. Esse quadro atual diverge do eixo ético-político da Reforma Psiquiátrica e, consequentemente, do próprio estatuto da SCI. Recuperar seus princípios tendo, como contexto, a atual direção tomada pela 
política de saúde mental no Brasil demonstra sua relevância. Seguindo, então essa perspectiva, o artigo apresentará os resultados obtidos no processo de implantação da SCI no CAPS-II Dr. Sergio Sibilio Fritsch a partir do marco teórico da psicanálise, sua importância como dispositivo de formação e qualificação dos profissionais da atenção psicossocial e os impasses que ocorreram por se tratar de um processo orientado pela psicanálise. Tais impasses verificam a avaliação conduzida por Zenoni (2000) a respeito de uma série de oposições entre atenção psicossocial e psicanálise: redução da pregnância do sintoma e emergência do inconsciente; promoção do bemestar e da saúde do usuário e emergência do desejo que pode acarretar mal-estar e angústia; resposta à demanda e interrogação da causalidade da demanda; defesa da unidade do usuário e localização da divisão do sujeito. Essas oposições, segundo a avaliação de Zenoni (2000), conduziriam o próprio psicanalista, a partir de um posicionamento crítico em relação à instituição, a oscilar entre a externalidade da psicanálise em relação à atenção psicossocial e sua internalidade como operadora de análise crítica da própria política de atenção psicossocial. No entanto, a leitura dos documentos da Reforma Psiquiátrica demonstra como a presença da psicanálise na atenção psicossocial contribui, em articulação com outras formaçóes profissionais (psiquiatria, psicologia, enfermagem, serviço social, fonoaudiologia, fisioterapia), para a ruptura com a autoridade psiquiátrica típica do modelo hospitalocêntrico e para a consolidação de uma abordagem multidisciplinar do sofrimento psíquico. $\mathrm{O}$ processo de implantação da SCI no CAPS-II Dr. Sergio Sibilio Fritsch a partir do marco teórico da psicanálise, que será apresentado nesse artigo, indica um diálogo difícil, mas não impossível, com a equipe, no sentido de se construir um espaço de construção do caso clínico que privilegie o discurso do usuário em sofrimento psíquico e assegure a circulação democrática do saber.

O artigo observa a Resoluçáo do Conselho Nacional de Saúde, no 510, de 07 de abril de 2016, referente ao registro de pesquisas no sistema CEP/CONEP (BRASIL, 2016). Por não conter e nem se referir a conteúdos de fala dos participantes da SCI, por não expor trechos de estudo de caso clínico, ele se enquadra especialmente no art.1, parágrafo VII, da Resolução no 510 , que dispóe que a pesquisa que objetiva o aprofundamento teórico de situaçôes que emergem espontânea e contingencialmente na prática profissional - desde que não revelem dados que possam identificar o sujeito - dispensa registro e avaliação pelo sistema CEP/CONEP. 


\section{Condições de implantação da SCI em Volta Redonda}

O município de Volta Redonda está localizado na Região do Médio Paraíba, no Estado do Rio de Janeiro. Em 2011, tinha uma população de 257.966 habitantes, correspondendo a 30,15\% do total da regiáo. Em 2015, a população estimada foi de 262.970 habitantes (IBGE, 2015).

A reforma psiquiátrica ocorreu no município no ano de 1994, com a intervenção do poder público municipal no único hospital psiquiátrico e de caráter privado: a Casa de Saúde de Volta Redonda (CSVR). O processo de intervenção obedecera à orientação mais geral da reforma psiquiátrica brasileira, de reestruturação da prevenção, acolhimento e cuidado com o sujeito em sofrimento psíquico grave e, consequentemente, da própria reestruturação do lugar de autoridade do saber psiquiátrico na abordagem e acolhimento do sofrimento psíquico. Em especial, o processo de intervenção na CSVR se localizava em um momento político da reforma psiquiátrica concentrado na tarefa de redução de leitos em hospitais psiquiátricos - o que Delgado (2013) denominou de desospitalização saneadora, objetivando a construção de um espaço multidisciplinar aberto à contribuição de diversos saberes.

Tradicionalmente, a CSVR era o lugar da recepção e cuidado do sofrimento psíquico, sendo responsável pela internação e tratamento desses casos, seguindo o modelo hospitalocêntrico de tratamento por meio da exclusão social por confinamento e da contenção física e medicamentosa. Dessa forma, o processo de reforma introduziu uma ruptura importante em relação à organização da política de saúde mental, no município, em torno do hospital e da hegemonia do saber psiquiátrico. E, de fato, no marco da intervenção da CSVR, o município começara a aderir a um modelo mais humanizado de abordagem e acolhimento do sofrimento psíquico, em acordo com a nova política pública de saúde mental implementada pela reforma psiquiátrica e com a adoção de procedimentos clínicos de abordagem e acolhimento do sofrimento psíquico orientados pela multidisciplinaridade.

A fundação do CAPS Usina dos Sonhos, no anexo à CSVR, em parte consolidou esse processo, substituindo a metodologia de tratamento oferecida pela arquitetura manicomial da CSVR. Além disso, verificaram-se as iniciativas do poder público municipal, da época, em investir na qualificação profissional de profissionais e gestores da rede de saúde mental do município através do Instituto Franco Basaglia 
e da inauguração do Centro de Estudos em Saúde Mental de Volta Redonda (CESAM), em 1994. O objetivo do CESAM era proporcionar, aos profissionais da saúde mental e à comunidade acadêmica de Volta Redonda, a investigação de temas associados ao campo da Reforma Psiquiátrica a fim de aprimorar e aprofundar as concepções teóricas que fundamentavam o processo de mudança na abordagem, acolhimento e tratamento do sofrimento psíquico.

Esse objetivo de formação e capacitação do CESAM levou à formação de uma parceria com a Sociedade Barramansense de Ensino Superior (SOBEU) para formalização de estágio dos estudantes de graduação nos equipamentos de saúde mental do município. No entanto, o processo de reforma psiquiátrica demonstrava, ainda, sua dependência do contexto político de época.

A posse, em 1997, de uma nova gestão na prefeitura teve impacto desfavorável na política de saúde mental do município, com consequências sobre o funcionamento do CAPS Usina dos Sonhos: manutenção de seu endereço no espaço asilar da CSVR, rotatividade elevada dos profissionais não médicos, sobrecarga de trabalho concentrada nesses profissionais e diminuição da carga horária da psiquiatria. E tudo isso acontecera em um contexto em que duas portarias ministeriais já haviam sido publicadas pelo Ministério da Saúde: a Portaria no 189/1991, que definira o financiamento público para os Núcleos/Centros de Atenção Psicossocial (NAPS/ CAPS), contemplando o custeio da assistência de pacientes que demandavam cuidados intensivos por equipes multiprofissionais; e a Portaria no 224/1992, que formalizara as funçôes dos NAPS/CAPS, sua composição básica e a amplitude de serviços à população.

As modificações na inserção dos profissionais da saúde mental promovida pela prefeitura em 1997 contrastavam com o processo de reforma psiquiátrica ao:

1. inflacionar a demanda de tratamento, por parte de usuários, no CAPS Usina dos Sonhos, com declínio da qualidade de atendimento, paralelamente à reduçáo da quantidade de profissionais, culminando com o encerramento da demanda espontânea (triagem de primeira vez);

2. preservar as atividades da CSVR mantendo ali o CAPS Usina dos Sonhos - o que evidenciou a resistência na política de saúde mental do município de avançar na direção de superar o modelo hospitalocêntrico e consolidar o modelo de atenção psicossocial. 
O estudo recente de Soares Martins (2017) apresenta o esquema de funcionamento do Serviço de Saúde Mental em Volta Redonda, entre os anos de 1995 e 1999, reproduzido na figura 1 .

Figura 1. Estrutura de Funcionamento do Serviço de Saúde mental em Volta Redonda $(1995-1999)$

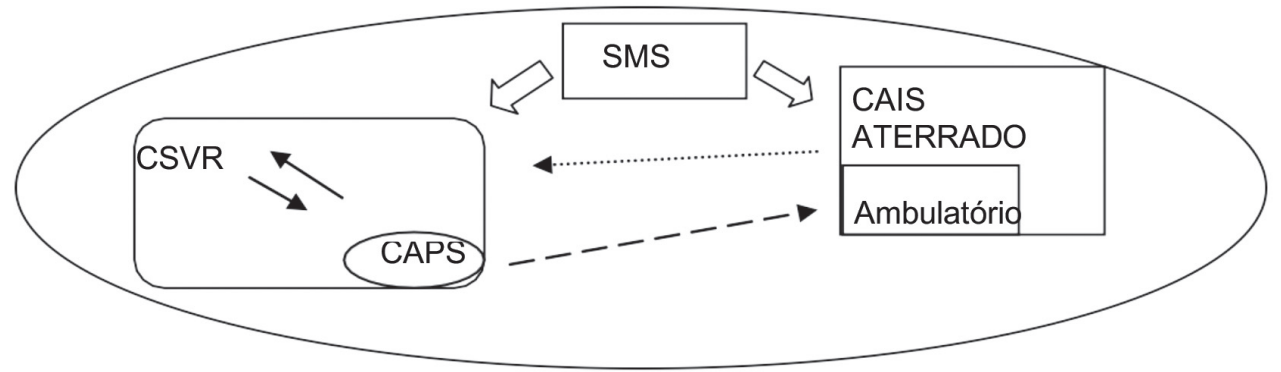

Fonte: Soares Martins (2017. p. 72)

As setas que partem da Secretaria Municipal de Saúde (SMS) indicam que os equipamentos de saúde mental estavam sob sua gestão; as setas de linha contínua designam o fluxo intenso entre a CSVR e o CAPS Usina de Sonhos; a seta tracejada indica fluxo intermitente de usuários não egressos da CSVR, para atendimento ambulatorial; a seta pontilhada indica fluxo recorrente do Hospital CAIS Aterrado e do ambulatório para a CSVR. Observando a figura 1, compreende-se o reforço da lógica ambulatorial e de internação típica do modelo hospitalocêntrico, pela organizaçáo do fluxo dos usuários, pela ausência de um plano estruturado de saúde mental para o município, pela não territorialização dos serviços e pela relação de complementaridade entre o CAPS e a CSVR (SOARES MARTINS, 2017). Essa organização contrastou, ainda, com a Emenda Constitucional 29/1999, que garantia que União, estados e municípios aplicassem, anualmente, em açôes e serviços públicos de saúde recursos próprios mínimos derivados da aplicação de percentuais calculados segundo acordos pactuados. No marco desta emenda, havia se verificado, nacionalmente, o aumento de $333 \%$ no financiamento em equipamentos substitutivos de saúde mental por habitante e a redução em 33\% dos recursos para equipamentos hospitalares de saúde mental, conforme mostra a tabela 1 . 
Tabela 1. Despesas per capita com Saúde Mental do Ministério da Saúde - Brasil - 2002 e 2009 (em R\$ de 2002)

\section{Descritor}

20022009 Variação 2002-2009

Despesa per capita com CAPS e outros dispositivos

$0,90 \quad 3,90$

$333 \%$

não hospitalares

Despesa per capita com açóes e programas hospitalares $\quad 2,77 \quad$ 1,86

$-33 \%$

Fonte: Costa et al. (2011, p. 4608).

É nesse quadro de contraste, da implantação de uma nova política nacional de saúde mental com a gestão da Prefeitura de Volta Redonda, entre o final dos anos de 1990 até o ano de 2016, que a rede de saúde mental se estrutura no município dispondo de dez equipamentos: CAPS Vila Santa Cecília; CAPS Usina de Sonhos; CAPS Dr. Sérgio Sibilio Fritsch; CAPSi Viva Vida (Infância e Adolescência); CAPS-ad Lúcia Maria Bessada; CAIS-Aterrado - Leitos de Agudos (transtorno mental e desintoxicação de álcool e outras drogas); Serviço de Residência Terapêutica - Vila Santa Cecília; Serviço de Residência Terapêutica Jardim Tiradentes; Serviço de Residência Terapêutica - Casa de Pedra; Serviço de Residência Terapêutica - São Luiz.

Em 2011, a área técnica de saúde mental de Volta Redonda endereçou a uma das autoras do presente artigo, docente e pesquisadora da universidade pública instalada no município de Volta Redonda (Regiáo Sul do Estado do Rio de Janeiro), o interesse em formalizar parceria para condução de processos formadores junto aos profissionais do CAPS-II Dr. Sergio Sibilio Fritsch e para formalização do estágio curricular do curso de graduação em psicologia, na área de psicanálise e saúde mental.

O trabalho teórico em psicanálise e saúde mental, preparatório para a elaboração do projeto de estágio curricular para o curso de Psicologia da universidade, a leitura da Política Nacional de Humanização da Atenção e Gestão do SUS (MINISTÉRIO DA SAÚDE, 2009), bem como o contato preliminar estabelecido com o horizonte da reforma psiquiátrica no município (a rotina de funcionamento da atenção psicossocial em um quadro de escassez de processos de formação e qualificação e a raridade do dispositivo de SCI) colocaram a seguinte pergunta: será que os equipamentos de saúde mental do município contribuem para o avanço da reforma psiquiátrica no município ou preservam o núcleo hospitalocêntrico, tal como se verificara no final da década de 1990 ? 
Com base nessa pergunta, foi elaborado o projeto de extensão, registrado na Plataforma SIGPROJ/MEC, com o objetivo de formalizar, em parceria com a universidade, um programa de formação e qualificação dos profissionais que trabalhavam na rede de saúde mental de Volta Redonda por meio da consolidaçáo do dispositivo da SCI no CAPS II - Dr Sergio Sibilio Fritsch. O problema da estruturação do processo de reforma psiquiátrica no município orientou, então, a elaboração do projeto obedecendo aos seguintes eixos:

1. O processo de Reforma Psiquiátrica no Brasil, que resultou na formulação e promulgação da Lei 10.216/2001.

2. A recuperação histórica do processo de Reforma Psiquiátrica ocorrido no município e que culminou com a intervenção do poder público municipal na CSVR, no ano de 1994, com o levantamento de 40 fotografias referentes às instalaçóes da Casa de Saúde de Volta Redonda, antes da intervenção (com o detalhamento da estrutura manicomial - celas-forte, camisas de força - do estado de conservação de enfermarias e da hotelaria indispensável ao hospital) e no processo de intervenção até a criação do CAPS Usina dos Sonhos.

3. A elaboração da supervisão clínica com base em conceitos psicanalíticos, e tendo como base os seguintes pontos: a localização do saber teórico na clínica do CAPS, a universidade como parceira e a SCI no horizonte da Reforma Psiquiátrica.

A SCI foi implementada no início de 2012, a partir da aprovação do projeto de extensão da Plataforma SIGPROJ/MEC, e obtenção de bolsas anuais de extensão pelos editais Bolsa de Extensão 2012 (protocolo 98577.416.108833.29012012), Seleção Interna UFF/Proext 2013 (protocolo 110947.482.108833.27032012), Edital Bolsa de Extensão 2014 (protocolo 171077.781.108833.06022014). Foi finalizada em 2015. Durante os três anos ela ocorreu distribuída, quinzenalmente, na quarta-feira.

Participavam em torno de dez a 12 profissionais do CAPS com frequência variada: 1 da coordenação, 2 técnicos da limpeza, 2 psicólogos, 1 enfermeiro, 2 cuidadores, 1 fisioterapeuta, 1 fonoaudióloga, 1 oficineira e, eventualmente, 1 psiquiatra. Além dos profissionais do CAPS, participavam também os estudantes do curso de Psicologia que integravam o projeto de extensão e, eventualmente, a coordenadora da área técnica de saúde mental do município. Os estudos de caso, conduzidos por Nishimura (2014) e Barros Lima (2016) tornaram público o processo de implantação da SCI no CAPS. 


\section{Metodologia}

O projeto adotou, para a elaboração do programa de formação e qualificação dos profissionais que trabalhavam na rede de saúde mental de Volta Redonda, a metodologia qualitativa de construção do caso clínico, tal como fora definido por Figueiredo (2004) e Viganó (2010). Tal metodologia fora assim denominada para designar a especificidade do método de pesquisa em psicanálise em relação à formalização empírica idealizada pela ciência positivista e cujo princípio é a supressão da variável sujeito em nome da objetividade. Neste sentido, a construção do caso clínico tem, como base, a elaboração dinâmica de um saber cujo foco é o sujeito possibilitando a inteligibilidade do caso clínico em saúde mental por meio da determinação da marca do caso e de sua construção. É justamente a valorizaçáo da marca do caso que sustentou a relevância da construção do caso clínico como metodologia do projeto de extensão.

No que se refere à preparação do material sobre a Reforma Psiquiátrica, a metodologia de construção do caso clínico tomou, como sujeito, o Município de Volta Redonda, e orientou o levantamento e a análise dos documentos direta e indiretamente ligados a ela. O estudo de caso conduzido por Nishimura (2014) representa a síntese trazida a público deste levantamento. Dessa forma, a preparação do material se valeu do estudo de caso de Nishimura (2014), destacando para os profissionais o conjunto de documentos que dispóe sobre o processo de reforma psiquiátrica e sobre a legislação de CAPS.

Outro aspecto relativo ao emprego da metodologia de construçáo do caso clínico se refere à apresentação dos casos, pelos profissionais do CAPS-II, na rotina da supervisão clínica. Tal metodologia possibilitou a inteligibilidade do caso clínico em saúde mental, tomando como base os seguintes eixos:

1. A reforma psiquiátrica, sua relevância na emergência de um novo modelo que responda efetivamente ao sofrimento psíquico associado aos quadros de psicose (hipocondria, automutilação, errância, estupor catatônico, declínio do sentimento de vida, passagem ao ato) e a concepção de usuário, que daí advém: cabendo à SCI debater junto com os membros da equipe do CAPS-II as noçôes de cidadania e autonomia como operadores desta nova concepçáo e garantidoras de inclusão social. 
2. A marca da equipe de cada CAPS: organização interna, dificuldade na consolidação do sistema de acolhimento do usuário a partir da referência à rede, relação com a gestão, sobrecarga de trabalho, orientaçôes teóricas, dificuldades com os casos, relação com o saber psiquiátrico - cabendo à supervisão o trabalho de integrar os profissionais em torno do conhecimento do processo de reforma psiquiátrica, do lugar que possuem nesse processo e, como a partir desse lugar, é possível abordar o caso clínico.

3. A concepção freudiana de sujeito à luz da sobredeterminação pela pulsão, pelo ato e pela transferência: o trabalho, na SCI, a partir da sobredeterminação do sujeito, constituiu o eixo teórico em torno do qual se deu a própria construção do caso:

a. No que se refere à pulsão, a ênfase da SCI recaiu sobre a possibilidade dos profissionais do CAPS-II reconhecerem, no conteúdo discursivo do usuário, a experiência mais radical da angústia e sua correlação com a desestabilização pulsional e a interrogarem sobre a posição do sujeito frente a ela (na psicose, o sujeito é reduzido à posição de objeto da angústia);

b. Em relação ao ato, a ênfase foi orientar os profissionais para reconhecer quando o ato tem a característica de tentar solucionar a invasão pulsional experienciada pela angústia ou quando o ato ocorre orientado pela angústia;

c. Quanto à transferência, o trabalho da SCI sustentou uma perspectiva da clínica que depende da relação transferencial estabelecida com os membros da equipe e ultrapassa os efeitos neurofisiológicos do psicofármaco prescrito.

4. O caso clínico: seguindo a fundamentação teórica da psicanálise, a construção do caso explorou os pequenos índices evidenciados na história clínica de cada sujeito em sofrimento psíquico e que não são contemplados pelo saber psiquiátrico exclusivamente centrado na classificação dos sintomas e prescrição de psicofármacos. Para isso, a SCI trabalhou o tripé conceitual, composto por sujeito, rede (de saúde, de outras políticas intersetoriais, familiar, social e cultural) e território (o lugar da vida do sujeito, as características culturais, o laço social). E o considerou como base para a própria elucidação do projeto terapêutico para cada caso. 


\section{Resultados e Discussão}

A partir da indissociabilidade entre ensino, pesquisa e extensão, que define a universidade, o processo de construção e condução da SCI no CAPS-II de Volta Redonda, a pergunta inicial do projeto de extensão - sobre o estado atual do processo de reforma psiquiátrica - ganhou a seguinte especificidade:

1. Em que momento da reforma psiquiátrica se encontra o município de Volta Redonda?

2. Que clínica fora possível, até aquele momento, no CAPS-II?

Conforme fora mencionado anteriormente, o contato preliminar à experiência da SCI revelava que o processo de reforma psiquiátrica náo funcionava bem: apenas o CAPSi Viva Vida (Infância e Adolescência) dispunha de SCI, além da raridade de processos de formação e qualificação profissional para as equipes da atenção psicossocial no município. Esses fatores explicam as seguintes características de funcionamento encontradas no CAPS-II: ateorismo, clínica do transtorno e polimedicação.

\section{Ateorismo, clínica do transtorno e polimedicação na atenção psicossocial}

Durante os primeiros encontros, observou-se a hegemonia do referencial da psiquiatria na compreensão do sofrimento psíquico, com a hipótese da causalidade neurofisiológica. A adoção desta hipótese por parte dos profissionais do CAPS-II apareceu na construção do caso clínico, por meio de sua organização em torno do múltiplo diagnóstico e da prescrição de diferentes psicofármacos, segundo uma direção que tendia a colocar como secundárias as noçôes de cidadania e autonomia que embasavam a concepção de usuário e que garantiam a inclusão social. De fato, durante as primeiras supervisões, foi possível identificar três efeitos decorrentes da presença hegemônica do saber psiquiátrico, sem uma problematização maior do conteúdo discursivo apresentado pelo usuário na rotina das atividades do CAPS-II:

1. O ateorismo na base da clínica ali exercida.

2. A proporcionalidade entre uma clínica do transtorno psiquiátrico e a prescrição de diferentes psicofármacos para um mesmo usuário em sofrimento psíquico: não raro um mesmo usuário em sofrimento psíquico apresentava múltiplos diagnósticos de transtornos e a prescrição de psicofármacos obedecia a essa proporcionalidade, sugerindo polimedicação. 
3. O desconhecimento por parte dos profissionais do equipamento de saúde mental sobre os efeitos das interações medicamentosas nos usuários e a inflação de terminologias imprecisas que localizavam, no próprio usuário, a causa e a evolução do sofrimento.

Esses efeitos, identificados localmente no CAPS-II ao longo do processo de consolidação da SCI, são tributários de um impasse histórico no campo do diagnóstico em psiquiatria. De um lado, a psiquiatria foi o campo de elaboração dos conceitos psicopatológicos que estão no fundamento do saber acumulado sobre o diagnóstico da loucura. De fato, para a psiquiatria situada entre o fim do século XIX e o início do século XX, decifrar a experiência da loucura era observá-la, descrevê-la e classificá-la em um corpus simples e que se acumulava nos manuais de psicopatologia. De outro, a construção de grandes sistemas diagnósticos reconhecidos pela OMS - como, por exemplo, o Código Internacional de Doenças (CID-10) e o Manual Diagnóstico e Estatístico dos Transtornos Mentais (DSM) - acabou produzindo o divórcio dos referenciais teóricos da psicanálise e da fenomenologia que embasavam a pesquisa etiológica em psicopatologia; a ascensão da tendência ao ateorismo, à clínica do transtorno e à polimedicação.

No que se refere à postura ateórica desvinculada de qualquer debate conceitual em torno de marcos teóricos, as primeiras SCIs evidenciaram uma organização do CAPS-II, centrada na alternância entre uma estrutura igualitária e hierárquica (TEIXEIRA, 2007). A estrutura igualitária tendia a acirrar a rivalidade entre os membros da equipe com formaçóes distintas e hierárquicas, dissolvia as rivalidades na abordagem do caso clínico pela localização da psiquiatria como princípio normativo para discussão diagnóstica e rara interrogação a respeito da correlação entre os comportamentos observados em usuários que haviam passado por longas internaçôes psiquiátricas e a prescrição continuada de psicofármacos. Por outro lado, observou-se entre os profissionais o uso pouco rigoroso de conceitos da reforma psiquiátrica (vínculo, sujeito, autonomia, rede) e da psicopatologia (transtorno mental, psicose, desencadeamento), para se referir tanto ao quadro clínico apresentado pelos usuários em atendimento no CAPS-II quanto à relação com a rede e o território. Esse uso dos conceitos se refletia na adesão a uma terminologia imprecisa (por exemplo, resistente ao projeto terapêutico) que tendia a ontologizar 
os efeitos adversos da prescrição de psicofármacos, interpretando-os como típicos do comportamento de quem apresenta um transtorno mental.

Tal contrariedade entre o uso pouco rigoroso dos conceitos no exercício da clínica e os eixos da reforma psiquiátrica encontra sua base na tendência, observada no modelo da atenção psicossocial, à aversão a todo saber constituído sob a forma de teoria da clínica e à adoção de uma fenomenologia do cuidado e da reabilitação articulada ao ecletismo teórico, conforme foi constatada por autores brasileiros como Elia e Galvão (2000) e Campos (2008). Tais autores mostraram como a aversão ao saber e a ancoragem no ecletismo teórico deram origem a uma Babel epistemológica, fragilizando a abordagem multidisciplinar e contribuindo para a preservação de um princípio normativo com base no diagnóstico psiquiátrico. Campos (2008) especifica essa Babel, identificando, por exemplo, a presença de uma abordagem sistêmica sob o rótulo de reabilitação psicossocial ou de uma psicanálise "misturada" com psicofarmacologia psiquiátrica. E tanto Elia e Galvão (2000) como Campos (2008) afirmam que essa Babel acabou contribuindo para consolidar o CID e o DSM como referências exclusivas para estabelecimento de diagnóstico na atenção psicossocial.

O CAPS-II, onde se instalou a SCI, não se furtava a presença dessa Babel epistemológica. Ela apareceu na forma da objeção à psicanálise como referencial teórico, da direção teórico-clínica centrada no princípio de cuidado e reabilitação e da adesão ao saber psiquiátrico, vigorando a mistura entre cuidado, reabilitação e psicofarmacologia, bem como uma exacerbação da normatização pelo cuidado.

\section{O referencial da psicanálise na SCI}

A identificação dos três efeitos decorrentes da hegemonia do saber psiquiátrico (ateorismo, clínica do transtorno e polimedicação) foi o ponto a partir do qual foi implantada e conduzida a SCI durante os três anos de seu funcionamento, articulando-se às perguntas sobre o status da reforma psiquiátrica no município e sobre a concepção de clínica do CAPS-II.

Um dos pontos levantados foi se a urgência de diagnósticos e a polimedicação se localizavam lado a lado à experiência de insuportabilidade do sofrimento psíquico para o usuário e seus familiares e ao hiperconsumo de psicofármacos. De fato, verificou-se durante a SCI que o sofrimento era visto ora pelos usuários, ora pela família, ora por ambos, como uma ameaça que precisava ser suprimida o mais 
breve possível por meio do diagnóstico e da medicação. Particularmente, no CAPSII, apesar da abordagem humanizada para o sofrimento psíquico, preservava-se o entendimento de que se tratava de um problema médico e, consequentemente, gravitava em torno da psiquiatria.

O relato de experiência do psicanalista Marcelo Veras (2010), orientado pela presença da psicanálise na abordagem do sofrimento psíquico durante sua gestão como diretor da Colônia Juliano Moreira (Salvador - Bahia), mapeia a ocorrência de duas concepçóes do que vem a ser o atendimento clínico: uma, que segue a autoridade do saber psiquiátrico, estruturada pela lógica da identificação, e na qual as diretrizes do tratamento são previamente formatadas pelo saber especializado, e outra concepção, que segue a lógica da transferência segundo a psicanálise, e que considera a adesão do sujeito ao projeto terapêutico como contingencial, podendo, ou não, ocorrer. Veras (2010) deixa claro que a concepção orientada exclusivamente pela psiquiatria - mesmo no marco da reforma psiquiátrica - pode gerar o efeito colateral de exacerbar o resgate de princípios normatizadores por meio da valorização exclusiva de protocolos e projetos terapêuticos. O estudo de Amancio (2012), em torno de sua experiência como psicanalista em um CAPSi no Rio de Janeiro, segue a mesma linha de avaliação do impacto da organização da atenção psicossocial em torno da concepção da psiquiatria sobre o posicionamento ético dos profissionais, chamando a atenção para o fato de que a exacerbação de princípios normatizadores é inseparável do ateorismo que domina a formação dos profissionais na atenção psicossocial.

Considerando essas referências, a valorização do traço contingencial fundamentado no tripé conceitual composto pelo usuário, a rede e o território - foi fundamental para uma nova interpretação do texto da Portaria 1.174/2005, que dispóe sobre os objetivos da SCI. De fato, a marca da construção psicanalítica do caso clínico como metodologia da SCI foi a de pactuar com a equipe do CAPS-I a possibilidade de reconhecimento da conjuntura de desencadeamento para cada caso apresentado, de interrogação sobre a posição subjetiva do usuário - quando procura reparar o desencadeamento sofrido por meio do delírio ou quando está determinado por ele, conforme se verifica nas passagens ao ato. E se orientar na Portaria 1.174/2005, a partir da fundamentação desta dimensão contingencial no tripé usuário-rede-território, permitiu à supervisão identificar o momento em que o saber psiquiátrico se apresentava como princípio normativo da construção do caso e da elaboração do projeto terapêutico individual. 
A partir, então, da discussão do problema da relação entre diagnóstico psiquiátrico e direção de tratamento, a experiência da SCI avançou para problematizar, junto à equipe, a reforma psiquiátrica no município, interrogando especificamente sobre o entendimento do que vem a ser a clínica na atenção psicossocial em um quadro de ateorismo ou ecletismo teórico. Para isso, avaliou a demanda social, constatando o endereçamento ao CAPS-II de formas clínicas de sofrimento psíquico, cujo diagnóstico apresenta-se atualmente de forma nebulosa, com difícil elucidação quanto à conjuntura de desencadeamento na vida do sujeito: hegemonia de passagens ao ato, do sentimento de vida em declínio, da alienação a uma terminologia do saber psiquiátrico, que indica mais a pregnância da dimensão comportamental do sofrimento (por exemplo, sou borderline, sou bipolar) do que o funcionamento subjetivo mais singularizado.

Dessa forma, diante da apresentação clínica atual da psicose, os profissionais da equipe do CAPS-II sabiam descrever os comportamentos apresentados pelos sujeitos em tratamento, mas quando a SCI interrogava pelo funcionamento subjetivo, a resposta retornava na forma da adesão ao vocabulário da psiquiatria com referências às categorias do CID- 10 e do DSM.

Particularmente, abundava a ocorrência de sujeitos que padeciam de neologismos, fenômenos hipocondríacos, desestruturação do eu, delírios sem alucinaçôes, frases impostas - indicando o funcionamento subjetivo de uma psicose. No entanto, o convite ao aprofundamento do estudo da psicose era muitas vezes negligenciado em nome da mistura entre princípios de cuidado e categorias psiquiátricas - de modo que a clínica da psicose cedia para a redução a uma clínica do transtorno de personalidade, transtorno de humor, depressão e ascendia o saber psiquiátrico como princípio normatizador do diagnóstico e da elaboração do projeto terapêutico. Dessa forma, ao mesmo tempo em que as apresentaçóes de caso traziam evidência do funcionamento psicótico, traziam também a marca da diluição da complexidade do desencadeamento pelo seu entendimento como dificuldade de aderência ao projeto terapêutico.

A retomada da referência à psicanálise, ao longo da SCI, ganhou sua relevância nesse quadro de progressão da disciplina classificatória do sofrimento psíquico, em psiquiatria, e de inflação da normatização. O que levou a SCI a fazer um breve levantamento histórico das ediçôes do DSM, sintetizado na tabela 2, para apresentar aos profissionais do CAPS-II a progressão do número de classificaçôes diagnósticas. 
Tabela 2. Síntese das Ediçóes do DSM

\begin{tabular}{ccc}
\hline Ediçóes do DSM & Ano de Publicação & Categorias \\
\hline DSM-I & 1952 & 106 \\
DSM-II & 1968 & 182 \\
DSM-III & 1980 & 265 \\
DSM-III-TR & 1989 & 292 \\
DSM-IV & 1994 & 297 \\
DSM-IV-TR & 2000 & 374 \\
DSM-5 & 2013 & 300 \\
\hline
\end{tabular}

Fonte: Adaptado de Mayes e Horwitz (2005, p. 251).

Ressaltando a edição do DSM-III, a SCI expôs o quadro de transformaçôes históricas sofridas no campo da psiquiatria:

1. A American Psichiatry Association (APA) propóe uma mudança de paradigma importante na pesquisa do sofrimento psíquico de tal forma que já no DSM-III não se encontra mais a referência ao funcionamento subjetivo e aos fatores que contribuem para a origem do sofrimento, mas aos fatores comportamentais mais manifestos. Tomando como exemplo a depressão, observa-se o recuo das categorias clínicas diferenciais nas síndromes depressivas (depressão reacional, depressão neurótica, melancolia psicótica) e a ascensão da intensidade do humor e suas consequências (insônia, perda de energia, anedonia, sentimento de desvalorização) com afrouxamento da distinção dos limites entre depressão e tristeza, doença depressiva e estado de ansiedade, psicose afetiva e esquizofrenia.

2. Essa mudança de paradigma não se separa da intensificação do procedimento médico de prescrição de psicotrópicos: para Maleval (2012), por exemplo, a mudança de paradigma na psiquiatria abriu a brecha para os excessos no campo psicofarmacológico.

Foi possível trabalhar na supervisão, a base teórica do DSM-III: kraepeliniana, na medida em que define o sofrimento psíquico segundo o modelo da doença orgânica e, consequentemente, estabilizado por meio da prescrição de psicotrópicos. No que tange ao modelo kraepeliniano, foi fundamental discutir o valor científico do DSMIII com base no artigo científico de Wyatt (2013), que chama atenção para o fato 
de como, no marco dos anos 1990, os transtornos mentais foram cada vez mais medicalizados, localizando nesse fato uma simbiose entre psiquiatria e indústria farmacêutica. Wyatt (2013) posiciona essa simbiose como o motor de explicaçôes não científicas acerca da etiologia neurofisiológica dos transtornos mentais.

A preocupação em elencar, para os profissionais dos CAPS, os psicofármacos disponibilizados pela farmácia do SUS em Volta Redonda foi coerente com a preocupação em posicioná-los criticamente diante da contaminação da atividade clínica pela multiplicação dos diagnósticos e produção de sujeitos medicados sem a abordagem do sofrimento psíquico em sua dimensão subjetiva, assim como conscientizá-los para a importância da articulação entre uma teoria sobre o funcionamento subjetivo e a clínica do sofrimento psíquico.

Durante o processo de construção do estudo de caso, Barros Lima (2016) ressaltou a expansão da prática psiquiátrica - restrita, no século XIX, ao âmbito asilar - para um número cada vez maior de sujeitos, segundo o fenômeno de medicalização do social e sua consolidação como autoridade de saber para disciplinas com aplicabilidade na área da saúde e da saúde mental (Serviço Social, Psicologia, Fisioterapia, Fonoaudiologia). Sobre esse fenômeno, Barros Lima (2016) corroborou Lucchetti et al. (2010), que identificaram que uma parcela dos medicamentos prescritos no Brasil pertence à classe dos psicofármacos: benzodiazepínicos, antidepressivos, neurolépticos, anticonvulsivantes ou estimulantes do sistema nervoso central. E, não raras vezes, são prescritos por necessidade de controle do comportamento, depressão e transtornos do sono.

Ao longo dos processos formativos, na supervisão, foi possível pensar criticamente o horizonte da reforma psiquiátrica no município, colocando a questáo de que a formulação de novas classificações diagnósticas, aliada à expansão tecnológica no setor de psicotrópicos, não erradicou o modelo manicomial. Se, de um lado, a intervenção na CSVR defendeu a ruptura com esse modelo, com a consequente redução da oferta e ocupação de pacientes em leitos localizados em manicômio, por outro, entendeu-se que a expansão do uso de psicotrópicos para o tratamento das formas de sofrimento psíquico tenderia a substituir o manicômio, segundo o próprio exemplo de Delay e Deniker, que já em 1952 utilizavam a clorpromazina para conter as agitaçóes psicomotoras de pacientes com diagnóstico de esquizofrenia, objetivando assegurar uma enfermaria silenciosa (FERRAZZA, 2009). Tomando como referência os documentos da Reforma Psiquiátrica, observou-se que esse ponto 
contradizia suas diretrizes, expostas na I Conferência Nacional de Saúde Mental (BRASIL, 1987) e a orientação da Organização Mundial da Saúde quanto ao uso racional de medicamentos, ou seja, a prescrição de medicamentos observando as condiçôes clínicas de um usuário em sofrimento: doses adequadas às situaçôes clínicas por um período necessário ao tratamento e ao menor custo para si e para a comunidade (WHO, 2004).

O debate em torno da polimedicação e clínica do transtorno em um mesmo caso clínico, durante a SCI, exigiu recorrer aos documentos da Política Nacional de Medicamentos (PNM), aprovada pela Comissão Intergestores e pelo Conselho Nacional de Saúde, por meio da Portaria 3.916/1998. E, e a partir daí, estabelecer uma interrogação crítica do que se entende sobre a Relação Municipal de Medicamentos do SUS de Volta Redonda - REMUME (VOLTA REDONDA, 2013). A REMUME (2013) foi apresentada aos profissionais do CAPS-II em três partes: a relação municipal de medicamentos por ordem alfabética, disponíveis conforme unidade de dispensação; o anexo 1 -relação de medicamentos disponíveis pelo componente estratégico da assistência farmacêutica (CESAF); o anexo 2 relação de medicamentos disponíveis em unidades hospitalares (uso interno). Das 542 substâncias catalogadas no REMUME, foram identificados os psicotrópicos disponibilizados pela prefeitura, elencados na tabela 3 .

Tabela 3. Relação dos psicotrópicos distribuídos pela Prefeitura Municipal de Volta Redonda

\begin{tabular}{ll}
\hline Fármaco & Indicação \\
\hline Amitriptilina, cloridrato & Estados depressives; enurese noturna. \\
\hline Bupropiona, cloridrato & Depressão \\
\hline Buspirona & Ansiedade acompanhada ou não de depressão \\
\hline Carbamazepina & Epilepsia; bipolaridade; síndrome de abstinência alcoólica \\
\hline Carbonato de lítio & $\begin{array}{l}\text { Transtornos bipolares; prevenção da fase depressiva; } \\
\text { hiperatividade }\end{array}$ \\
\hline Clomipramina, cloridrato & $\begin{array}{l}\text { Estados depressivos; depressão associada à } \\
\text { esquizofrenia; síndromes depressivas; fobias; crises de pânico }\end{array}$ \\
\hline Clonazepam & Transtornos de ansiedade; epilepsia; depressão \\
\hline Clorpromazina, cloridrato & Psicose; ansiedade \\
\hline
\end{tabular}




\begin{tabular}{|c|c|}
\hline Fármaco & Indicação \\
\hline Diazepam & Estados de ansiedade; epilepsia \\
\hline Fenitoína sódica & Epilepsia \\
\hline Fenobarbital & Propriedades anticonvulsivantes \\
\hline Fluoxetina, cloridrato & Depressão associada ou não à ansiedade \\
\hline Haloperidol & Esquizofrenia; paranoia \\
\hline Haloperidol, decanoato & Psicose crônica \\
\hline Hidrato de cloral & Estados de ansiedade \\
\hline Imipramina & Estados depressivos \\
\hline $\begin{array}{l}\text { Imipramina, pamoato (tofranil } \\
\text { pamoato) }\end{array}$ & $\begin{array}{l}\text { Estados depressivos; depressão com distúrbios de } \\
\text { personalidade; transtorno do pânico }\end{array}$ \\
\hline Levomepromazina & Ansiedade; depressão; esquizofrenia \\
\hline Lorazepam & Ansiedade \\
\hline Memantina, cloridrato & Alzheimer \\
\hline Naloxona & Depressão respiratória \\
\hline Naltrexona & Antagonista opioide \\
\hline Nitrazepam & Distúrbios do sono \\
\hline Periciazina (neuleptil) & Distúrbios de caráter; autismo; hipermotividade \\
\hline Risperidona & Ampla gama da esquizofrenia \\
\hline \multirow[t]{2}{*}{ Sertralina } & Depressão acompanhada ou não de ansiedade \\
\hline & Transtorno-obsessivo-compulsivo; transtorno do pânico. \\
\hline Sulpirida & Esquizofrenia \\
\hline Tioridazilda & Esquizofrenia crônica \\
\hline Valproato de sódio ou ácido valpróico & Epilepsia \\
\hline Pipotiazina, palmitato & Psicose crônica; psiquiatria infantil \\
\hline Topiramato & Epilepsia \\
\hline Sulpirida & Esquizofrenia \\
\hline Tioridazilda & Esquizofrenia crônica \\
\hline
\end{tabular}

Fonte: Adaptado de Volta Redonda (2013). 
Conforme foi abordado anteriormente, nota-se uma grande defasagem do diagnóstico diferencial, defendido pela reforma psiquiátrica e pela psicanálise. Foi proposto aos profissionais do CAPS-II elaborar uma distinção entre os conceitos de mental e psíquico, situando o domínio de atuação da psiquiatria em torno do conceito de mental, associado ao orgânico e, portanto, divorciado de uma teoria do psíquico. Essa distinção objetivou apresentar a origem histórica da psiquiatria no modelo organicista, que concebe contemporaneamente o sofrimento psíquico como produto de alteraçóes neurofisiológicas e dispensa a psicanálise como referência para direção de tratamento desses casos.

A proposta de construção do caso clínico, durante a SCI, se posicionou na direção contrária da especialização do saber psiquiátrico e da normatização exacerbada. Situando o sujeito como variável fundamental, tomou-se como referência três eixos norteadores a partir da psicanálise:

1. O conhecimento da definição de sujeito com ênfase na sua sobredeterminação pela pulsão e pela transferência: no que se refere à pulsão, a ênfase foi a localização da experiência mais radical da angústia como expressão de invasão pulsional e a interrogação sobre a posição do sujeito frente a ela (quando procura reparar a invasão pulsional, por meio do delírio ou quando está determinada por ele, conforme se verifica nas passagens ao ato); em relação à transferência, a ênfase foi a necessidade de acolher a singularidade pela sustentação da relação transferencial, respeitando o limite de cada sujeito. $\mathrm{O}$ trabalho, na SCI, a partir da sobredeterminaçáo do sujeito constituiu o eixo em torno do qual se deu a própria construçáo do caso.

2. A dimensão contingencial de cada caso, que está intimamente articulado ao eixo da sobredeterminaçáo do sujeito: a conjuntura de vida que cerca o desencadeamento do sofrimento psíquico, sua relaçáo com a família, as instituições, a determinação das variáveis psíquicas de desestabilização no caso, a sucessão de tratamentos a que se submetera, os efeitos terapêuticos obtidos, e o procedimento profissional para a construção de uma proteção para o sujeito que permita o seu afastamento em relação à invasão de angústia verificada na psicose.

3. O saber dos profissionais sobre o contingencial de cada caso e o trabalho de articulação com a sobredeterminação do sujeito. 
A consideração desses eixos não objetivou uma oposição à prescrição de psicofármacos no CAPS-II. Mas sim trabalhar a função do medicamento, para cada caso, junto aos profissionais, usuários e familiares e sem dispensar a variável sujeito sobredeterminado pela pulsão. Afirmar o sujeito significou a possibilidade de interrogar no caso, e junto a cada usuário: o que cada um identifica como sendo sua conjuntura de desencadeamento e como se posiciona frente a ela; como cada um entende a medicação em suas vidas, que efeitos são identificados visando à construção de uma posição de autonomia frente ao ato do psiquiatra de prescrever psicotrópicos; o que é possível pactuar junto com a equipe do CAPS-II e com a rede de saúde de Volta Redonda em termos de laços sociais que não se apresentem como desencadeantes para o usuário.

Se a proposta da formação e qualificação dos profissionais de saúde mental do município de Volta Redonda por meio da SCI representou, inicialmente, um convite a constituir, na parceria com a universidade, um espaço de formação para a equipe do CAPS-II, ao final de seu período de funcionamento havia se consolidado como uma via possível para a construção do caso clínico e, consequentemente, para a emergência do sujeito nos marcos das noçôes de autonomia e cidadania, defendidas pela reforma psiquiátrica. ${ }^{1}$

\section{Referências}

AMANCIO, V. R. Uma clínica para o CAPS: a clínica da psicose no dispositivo da reforma psiquiátrica a partir da direção da psicanálise. Curitiba: CRV, 2012.

BARROS LIMA, M. No avesso da lógica do todo: uma experiência de clínica psicanalítica no campo da Saúde Mental. 2016. 110f. Trabalho de Conclusão de Curso (Graduação em Psicologia) - Universidade Federal Fluminense, Volta Redonda, 2016.

BRASIL. Ministério da Saúde. Conselho Nacional de Saúde. Resolução n. 510, de 07 de abril de 2016. Diário Oficial da União. Seção 1. n. 98, 24 maio 2016.

. Ministério da Saúde. I Conferência Nacional de Saúde Mental: Relatório Final. Brasília: MS, 1987.

CAMPOS, G. W. S. Produção de conhecimento, avaliação de políticas públicas em saúde mental: notas reflexivas. In: CAMPOS, R. O. et al. (Orgs.). Pesquisa avaliativa em saúde mental: desenho participativo e efeitos da narratividade. São Paulo: Aderaldo \& Rothschild, 2008, p. 97-102. 
COSTA, N. R. et al. Reforma psiquiátrica, federalismo e descentralização da saúde pública no Brasil. Ciência \& Saúde Coletiva. Rio de Janeiro, v. 16, n. 12, p. 4603-4614, 2011. Disponível em: http://www.scielo.br/scielo.php?script=sci_arttext\&pid=S1413-81232011001300009\&lng $=$ en\&nrm=iso>. Acesso em: $28 \mathrm{dez} .2018$.

DELGADO, P.G. Supervisão clínico-institucional: conceito e história. In: ESCOLA de Saúde Pública do Estado de Minas Gerais Augusto Nunes Filho (Org.). Supervisáo em saúde mental. Belo Horizonte: ESP-MG, 2013, p.18-27

ELIA, L.; GALVÃO, M. S. Estratégias de desconstrução da instituição fechada e produção de subjetividade. In CASTRO, N.; DELGADO, P. G. (Orgs.). De volta à cidadania: políticas públicas para crianças e adolescentes. Rio de Janeiro: Instituto Franco Basaglia, 2000. p. 71-93.

FERRAZZA, D. A Medicalização do social: um estudo sobre a prescrição de psicofármacos na rede pública de saúde. 2009. Dissertação (Mestrado em Psicologia) - Faculdade de Ciências e Letras, Universidade Estadual Paulista Júlio Mesquita Filho, Campus de Assis-SP, 2009.

FIGUEIREDO, A. C. A construção do caso clínico: uma contribuição da psicanálise à psicopatologia e à saúde mental. Revista latinoamericana de psicopatologia fundamental, v. 7,n. 1, p. 75-86,mar. 2004. Disponível em: http://www.scielo.br/scielo.php?script=sci_arttext\&pid=S1 4157142004000100075\&lng=en\&nrm=iso. Acesso em: 7 jan. 2019.

INSTITUTO BRASILEIRO DE GEOGRAFIA E ESTATÍSTICA. Coordenação de População e Indicadores Sociais. Síntese de indicadores sociais: uma análise das condiçôes de vida da população brasileira. Rio de Janeiro: IBGE, 2015.

LUCCHETTI, G. et al. Fatores associados ao uso de psicofármacos em idosos asilados. Revista de Psiquiatria do rio Grande do Sul, v. 2, n. 2, p. 38-43, 2010. Disponível em: http://www.scielo. br/pdf/rprs/v32n2/v32n2a03. Acesso em: 7 jan. 2019.

MALEVAL, J. C. Étonnantes mystifications de la psychotherapie autoritaire. Paris: Navarin, 2012.

MAYES, R.; HORWITZ, A. V. DSM-III and the revolution in the classification of mental illness. Journal of the History of the Behavioral Sciences, v. 1, n. 41(3), p. 249-267, 2005. Disponível em: https://www.researchgate.net/publication/7763046_DSM-III_and_the_revolution_in_ the_classification_of_mental_illness. Acesso em: 7 jan. 2019.

NISHIMURA, A. A evolução da política pública de saúde mental no Brasil: um estudo de caso sobre a Casa de Saúde de Volta Redonda (CSVR). 2014. 77 f. Trabalho de Conclusão de Curso (Graduação em Administração Pública) - Universidade Federal Fluminense, Volta Redonda, 2014.

SOARES MARTINS, G. C. O Centro de atenção psicossocial como espaço de afirmação do habitus profissional do enfermeiro na saúde mental do município de Volta Redonda. 2017. Tese (Doutorado em Enfermagem) - Programa de Pós-Graduação e Pesquisa da Escola de Enfermagem Anna Nery, Universidade Federal do Rio de Janeiro, Rio de Janeiro, 2017. 
TEIXEIRA, A. M. Tecendo a rede: a psicanálise na saúde mental. CliniCAPS. Impasses da Clínica, v. 1, n. 1, p. 1-11, 2007. Disponível em: https://www.clinicaps.com.br/clinicaps_pdf/ Rev_01/Revista01_art2_Antonio.pdf. Acesso em: 7 maio 2019.

VERAS, M. A loucura entre nós. Bahia: Aldeia Bahia Brasil, 2010.

VIGANÓ, C. A construção do caso clínico. Opção Lacaniana online Nova Série, v. 1, n.1, p. 1-9, 2010. Disponível em: http://www.opcaolacaniana.com.br/pdf/numero_1/A_construcao_ do_caso_clinico.pdf. Acesso em: 7 jan. 2019.

VOLTA REDONDA. Secretaria Municipal de Saúde. REMUME - Relação Municipal de Medicamentos do SUS de Volta Redonda. Volta Redonda: SMS-VR, 2013.

WORLD HEALTH ORGANIZATION. WHO model formulary 2004. Geneva: WHO, 2004. WYATT, J. W. Medicalization of depression, anxiety, schizophrenia, ADHD, childhood bipolar disorder and tantrums: scientific breakthrough, or broad-based delusion? Postępy Nauk Medycznych, t. XXVI, n. 1, p. 10-21, 2013. Disponível em: http://www.pnmedycznych.pl/wpcontent/uploads/2014/08/pnm_2013_010-021.pdf. Acesso em: 7 jan. 2019.

ZENONI, A. Psicanálise e instituição. A segunda clínica de Lacan. Abrecampos. Belo Horizonte, v. 1, p. 9-93, 2000.

\section{Agradecimentos}

À Pro-Reitoria de Extensão da Universidade Federal Fluminense (PROEX-UFF), pelo financiamento do Projeto de Extensão, por meio de bolsas de extensão, durante os três anos em que este esteve em vigor.

\section{Notas}

${ }^{1}$ C. H. de Lima elaborou o artigo. T. C. S. M. Sampaio revisou o texto e sugeriu correçôes, assim como na construção e formatação de tabelas e figuras. J. da S. Cunha colaborou na revisão do artigo, formatação de referências e elaboração do resumo. 


\section{Abstract}

Clinical-institutional supervision as a qualification device in Psychosocial Care: an experience of partnership with the University The conduct of clinical and institutional supervision is presented at a psychosocial care center located in the city of Volta Redonda, state of Rio de Janeiro, Brazil, resulting from an extension project that took place between 2012 and 2015, in a university located in same municipality. The process of psychiatric reform in the municipality is presented with two questions of the extension project: When is the psychiatric reform in Volta Redonda? What clinic had been possible so far at CAPS? From these questions, the process of clinicalinstitutional supervision was described, highlighting psychoanalysis as the main theoretical framework in the analysis of how the daily life of CAPS-II users lies on the horizon of psychiatric reform, on the role of psychiatry in the CAPS and in the construction of an alternative knowledge to psychiatry, recognizing the relevance of the theory, the subjective position of users and the knowledge of professionals. Finally, we highlight the relevance of the partnership with the university, as a space for formation and permanent critical reflection on the provision of mental health services within the psychiatric reform.

> Keywords: clinical-institutional supervision; psychosocial care; Psychiatric Reform; psychoanalysis; university. 